\title{
Stimulated Brillouin scattering in magnetized plasmas
}

Gert Brodin and Lennart Stenflo

\section{Linköping University Post Print}

\section{Tweet}

N.B.: When citing this work, cite the original article.

Original Publication:

Gert Brodin and Lennart Stenflo, Stimulated Brillouin scattering in magnetized plasmas, 2013, Journal of Plasma Physics, (79), 6, 983-986.

http://dx.doi.org/10.1017/S0022377813000664

Copyright: Cambridge University Press (CUP)

http://www.cambridge.org/uk/

Postprint available at: Linköping University Electronic Press

http://urn.kb.se/resolve?urn=urn:nbn:se:liu:diva-103069 


\title{
Stimulated Brillouin scattering in magnetized plasmas
}

\author{
G. B R O D I N ${ }^{1}$ and L. STE N F L O \\ ${ }^{1}$ Department of Physics, Umeå University, SE-901 87 Umeå, Sweden \\ (Gert.Brodin@physics.umu.se) \\ ${ }^{2}$ Department of Physics, Linköping University, SE-581 83 Linköping, Sweden
}

(Received 6 May 2013; revised 29 May 2013; accepted 29 May 2013; first published online 9 July 2013)

\begin{abstract}
Previous theory for stimulated Brillouin scattering is reconsidered and generalized. We introduce an effective ion sound velocity that turns out to be useful in describing scattering instabilities.
\end{abstract}

\section{Introduction}

Brillouin scattering instabilities are well known in the context of laser fusion (e.g. Kruer 1973; Tsytovich et al. 1973; Weiland and Wilhelmsson 1977; Rahman et al. 1981; Yadav et al. 2008; Simon 1995; Panwar and Sharma 2009). Mendonca (2012, review paper) has, in addition, examined such backscattering instabilities of electromagnetic beams carrying orbital angular momentum and stressed their relevance for plasma diagnostics.

In the 1970s it was predicted that the threshold values for stimulated Brillouin scattering can also be exceeded in ionospheric experiments (see the review papers Stenflo 2004; Gurevich 2007). The scattering by ion-cyclotron waves (e.g. Shukla and Tagare 1979; Samimi A. et al. 2013) and drift waves (Shukla et al. 1984) can then be important. Related experiments have later been outlined for piezoelectric semiconductor plasmas (e.g. Amin 2010).

In the present paper we are going to further extend the results of previous authors. Let us therefore start with the equation (Stenflo 1981; Shukla and Stenflo 2010)

$\frac{1}{\chi_{e}(\omega, \mathbf{k})}+\frac{1}{1+\chi_{i}(\omega, \mathbf{k})}=\frac{k^{2}\left|\mathbf{k}_{s-} \times \mathbf{u}_{0}\right|^{2}}{k_{s-}^{2} D_{s-}}+\frac{k^{2}\left|\mathbf{k}_{s+} \times \mathbf{u}_{0}\right|^{2}}{k_{s+}^{2} D_{s+}}$,

where $\chi_{e}(\omega, \mathbf{k})$ and $\chi_{i}(\omega, \mathbf{k})$ are the standard lowfrequency electron and ion susceptibilities for ionacoustic and electrostatic ion-cyclotron waves, $\omega$ and $\mathbf{k}$ are the frequency and wave vector, $\mathbf{k}_{s \pm}=\mathbf{k} \pm \mathbf{k}_{0}$ is the wave vector of the upper and lower sideband, respectively, $\mathbf{k}_{0}$ is the wave vector of the high frequency electromagnetic pump wave, $\mathbf{u}_{0}=e \mathbf{E}_{0} / m_{e} \omega_{0}$ is the electron quiver velocity of the pump with the electric field $\mathbf{E}_{0}$ and the frequency $\omega_{0}=\left(k_{0}^{2} c^{2}+\omega_{p e}^{2}\right)^{1 / 2},-e$ is the electron charge, $m_{e}$ is the electron mass, $c$ is the speed of light in vacuum and $\omega_{p e}$ is the electron plasma frequency. Furthermore, $D_{s \pm}=k_{s \pm}^{2} c^{2}-\omega_{s \pm}^{2}+\omega_{p e}^{2}$ in the absence of dissipation, where $\omega_{s \pm}=\omega \pm \omega_{0}$. For $\omega \ll \omega_{0}$ we have $D_{s \pm} \simeq \mp 2 \omega_{0}\left(\omega-\delta_{ \pm}\right)$, where $\bar{\delta}_{ \pm}=\mathbf{k} \cdot \mathbf{v}_{g} \pm k^{2} c^{2} / 2 \omega_{0}$, and $\mathbf{v}_{g}=\mathbf{k}_{0} c^{2} / \omega_{0}$.
Introducing $\varphi_{ \pm}$as the angle between $\mathbf{k}_{s \pm}$ and $\mathbf{u}_{0}$, we note that (1.1) can be simplified to

$$
\begin{aligned}
& \left(\frac{1}{\chi_{e}(\omega, \mathbf{k})}+\frac{1}{1+\chi_{i}(\omega, \mathbf{k})}\right)\left(\left(\omega-\mathbf{k} \cdot \mathbf{v}_{g}\right)^{2}-k^{4} c^{4} / \omega_{0}^{2}\right) \\
& \quad=\frac{k^{4} c^{2}\left|u_{0}\right|^{2} \sin ^{2} \varphi}{\omega_{0}^{2}}
\end{aligned}
$$

provided $\varphi_{+} \simeq \varphi_{-}=\varphi$, which, for example, holds for $|\mathbf{k}| \ll\left|\mathbf{k}_{0}\right|,\left|\mathbf{k}_{0}\right| \ll|\mathbf{k}|$ or if the wave vectors are parallel. Equation (1.2) is in agreement with Drake et al. (1974, Eq. (44)) in the unmagnetized limit. They used this formula to deduce the growth rate for modulational instabilities. Furthermore, four-wave interaction has been studied in a magnetized plasma for a one-dimensional geometry (Stenflo 1978), generalized to include the effects of a very large pump amplitude, also allowing for $\left|u_{0}\right|>\omega_{0} / k_{0}$, which is not within the regime of validity of (1.2). The advantage with our formulas below is their applicability to a general three-dimensional geometry spanned by the wave vectors and the external magnetic field.

\section{Derivations and results}

Next we assume that the interaction with the lower sideband $\left(\omega_{s-}, \mathbf{k}_{s-}\right)$ is dominant over the upper sideband $\left(\omega_{s+}, \mathbf{k}_{s+}\right)$, i.e. $\left|D_{s-}\right| \ll\left|D_{s+}\right|$. Rewriting (1.1) keeping only the interaction with the dominant sideband and assuming $\omega \ll \Omega_{e}$ and $\omega \gg k V_{T i}$, where $\Omega_{e}$ is the electron cyclotron frequency and $V_{T i}$ is the ion thermal velocity, we obtain (Shukla and Stenflo 2010)

$$
\begin{aligned}
& \left(\omega-\delta_{-}\right)\left(\omega^{4}-\omega^{2} \Omega_{I C}^{2}+k_{z}^{2} C_{s}^{2} \Omega_{i}^{2}\right) \\
& =\frac{\left(\Omega_{i}^{2} k_{z}^{2}-\omega^{2} k^{2}\right)}{2 \omega_{0}} \omega_{p i}^{2}\left|u_{0}\right|^{2} \sin ^{2} \varphi,
\end{aligned}
$$

where $\Omega_{I C}^{2}=\Omega_{i}^{2}+k^{2} C_{s}^{2}, \Omega_{i}$ is the ion-cyclotron frequency, $C_{s}$ is the ion sound velocity, $k=\left(k_{\perp}^{2}+k_{z}^{2}\right)^{1 / 2}$ and the subscripts $\perp$ and $z$ respectively stand for components perpendicular and parallel to the external magnetic field $\mathbf{B}_{0}=B_{0} \hat{\mathbf{z}}$. Here $\varphi$ is the angle between $\mathbf{k}_{s-}$ and $\mathbf{u}_{0}$. Then we consider the parametric decay instability and thus 


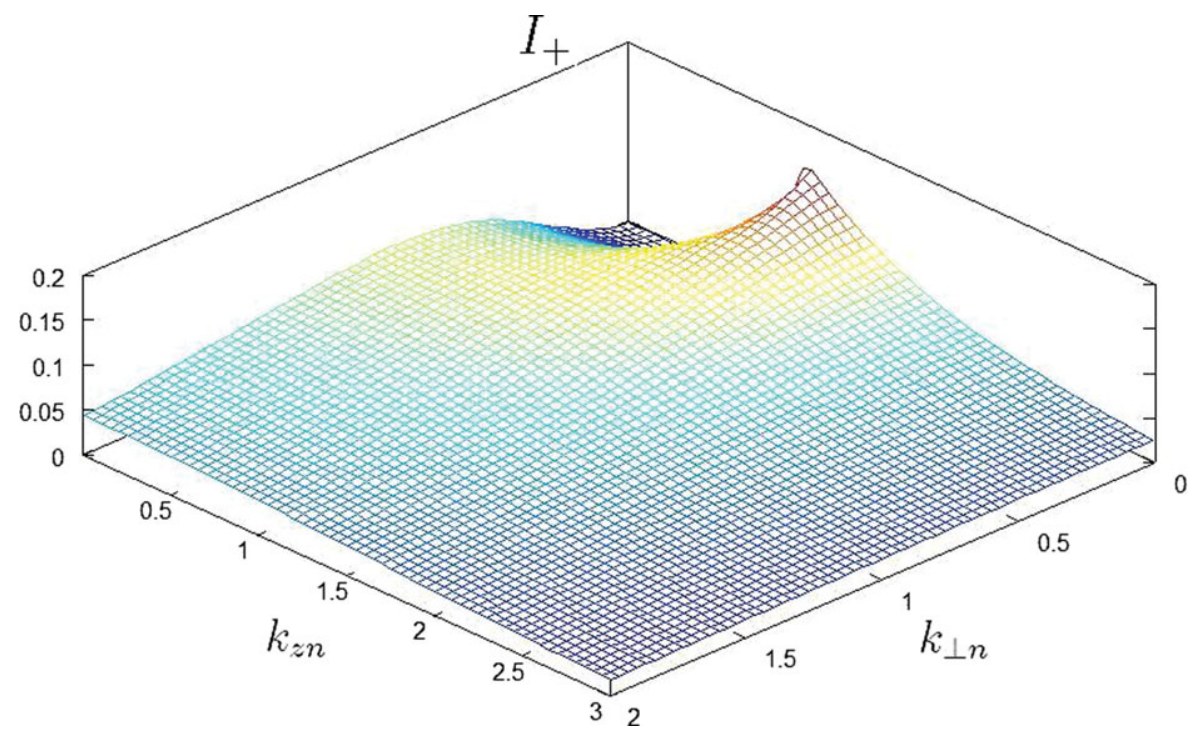

Figure 1. (Colour online) $I_{+}$plotted as a function of normalized wavenumbers $k_{z n}$ and $k_{\perp n}$.

divide the frequency into its real and imaginary parts, that is $\omega=\omega_{r}+i \gamma$. Furthermore, we take the sideband to be resonant, i.e.

$$
\omega-\delta_{-}=i \gamma
$$

such that $\omega_{r}=\delta_{-}$. Similarly, the low-frequency dispersion relation is supposed to be fulfilled, i.e.

$$
\omega_{r}^{4}-\omega_{r}^{2} \Omega_{I C}^{2}+k_{z}^{2} C_{s}^{2} \Omega_{i}^{2}=0,
$$

in which case the growth rate is

$$
\gamma^{2}=\frac{\left(\omega_{r}^{2} k^{2}-\Omega_{i}^{2} k_{z}^{2}\right)\left|u_{0}\right|^{2} \omega_{p i}^{2} \sin ^{2} \varphi}{4\left(2 \omega_{r}^{3}-\omega_{r} \Omega_{I C}^{2}\right) \omega_{0}},
$$

where the right-hand side is a positive definite factor because of the condition (2.3). Next we are interested in finding the fastest growing decay products. For this purpose we introduce the azimuthal angle $\phi$ for $\boldsymbol{k}$, i.e. we write $\boldsymbol{k}=k_{\perp}(\hat{\mathbf{x}} \cos \phi+\hat{\mathbf{y}} \sin \phi)+k_{z} \hat{\mathbf{z}}$. If we let $\mathbf{k}_{0}=k_{0} \hat{\mathbf{x}}$, then the resonance condition for the sideband (2.2) is fulfilled for $\cos \phi \simeq k^{2} / 2 k_{0} k_{\perp}$. With the azimuthal angle determined, we are free to vary $k_{z}$ and $k_{\perp}$ to maximize $\gamma$. Writing

$$
\gamma^{2}=I_{ \pm}\left(k_{z}, k_{\perp}\right) \frac{\left|u_{0}\right|^{2} \omega_{p i}^{2} \Omega_{i} \sin ^{2} \varphi}{2 C_{s}^{2} \omega_{0}},
$$

where $I_{ \pm}=\left(\omega_{r \pm}^{2} k^{2} C_{s}^{2}-\Omega_{i}^{2} k_{z}^{2} C_{s}^{2}\right) /\left(4 \omega_{r \pm}^{3}-2 \omega_{r \pm} \Omega_{I C}^{2}\right) \Omega_{i}$ and here the index \pm refers to the two roots of (2.3) given by $\omega_{r \pm}^{2}=\left(\Omega_{I C}^{2} / 2\right) \pm \sqrt{\left(\Omega_{I C}^{4} / 4\right)-k_{z}^{2} C_{s}^{2} \Omega_{i}^{2}}$, we thus want to find the maximum of $I_{ \pm}\left(k_{z}, k_{\perp}\right)$. In Figs. 1 and $2, I_{+}\left(k_{z}, k_{\perp}\right)$ and $I_{-}\left(k_{z}, k_{\perp}\right)$ are shown as functions of the normalized wavenumbers $k_{z n}=k_{z} C_{s} / \Omega_{i}$ and $k_{\perp n}=k_{\perp} C_{s} / \Omega_{i}$. We see that the fastest growing modes occur for the negative root and for both $k_{z n} \ll 1$ and $k_{\perp n} \ll 1$ in which case $I_{-}$approaches 0.5 (Fig. 2). The other mode (Fig. 1) has $I_{+}$always smaller than 0.2 and has a peak value for $k_{\perp n} \ll 1$ and $k_{z n}$ slightly larger than unity. Thus, the parametric decay instability will mainly occur for the negative root and with parallel and perpendicular wavelengths much longer than the effective gyro radius $C_{s} / \Omega_{i}$. Since the maximum occurs for small $k$, it is always possible to fulfill the condition, $\cos \phi \simeq k^{2} / 2 k_{0} k_{\perp}$ (for larger $k$ this condition may lead to $\cos \phi>1$, in which case the sideband resonance condition cannot be fulfilled simply by varying the azimuthal angle). Furthermore, for small $k$ the factor $\sin ^{2} \varphi$ in (2.5) is also maximized as we get $\sin ^{2} \varphi \simeq 1$.

For a strong pump wave $u_{0}$, the growth rate $\gamma$ found in (2.4) may be comparable to $\omega_{r}$. If $\delta_{-}<\delta_{+}$, we may still use (2.1), but naturally (2.2) and (2.3) cannot be applied for such a large pump amplitude. To study this case, we focus on the regime where $\omega \ll \delta_{-}$, in which case (2.1) can be written as

$$
\begin{aligned}
& \omega^{4}+\left(\Omega_{i}^{2} k_{z}^{2}-\omega^{2} k^{2}\right)\left(C_{s}^{2}+\frac{\sin ^{2} \varphi}{\left(2 \mathbf{k} \cdot \mathbf{k}_{0}-k^{2}\right) c^{2}}\left|u_{0}^{2}\right| \omega_{p i}^{2}\right) \\
& -\omega^{2} \Omega_{i}^{2}=0 .
\end{aligned}
$$

Interestingly, this is exactly the same dispersion relation as the usual one (i.e. (2.3)) for the linear low-frequency mode, except that the ion-sound velocity is now substituted according to $C_{s}^{2} \rightarrow C_{\text {eff }}^{2}$, where the effective ion-sound velocity is given by

$$
C_{\mathrm{eff}}^{2}=C_{s}^{2}+\frac{\sin ^{2} \varphi}{\left(2 \mathbf{k} \cdot \mathbf{k}_{0}-k^{2}\right) c^{2}}\left|u_{0}^{2}\right| \omega_{p i}^{2} .
$$

In order to get instabilities we must have $C_{\text {eff }}^{2}<0$ which in turn requires $\left(2 \mathbf{k} \cdot \mathbf{k}_{0}-k^{2}\right)<0$, which is fulfilled for a broad set of parameters. The solutions can thus be written as

$$
\omega_{ \pm}^{2}=\frac{\Omega_{\mathrm{eff}}^{2}}{2} \pm \sqrt{\frac{\Omega_{\mathrm{eff}}^{4}}{4}-k_{z}^{2} C_{\mathrm{eff}}^{2} \Omega_{i}^{2}}
$$

where $\Omega_{\mathrm{eff}}^{2}=\Omega_{i}^{2}+k^{2} C_{\mathrm{eff}}^{2}$. While the cyclotron frequency may have a stabilizing influence for small $k$, it is clear 


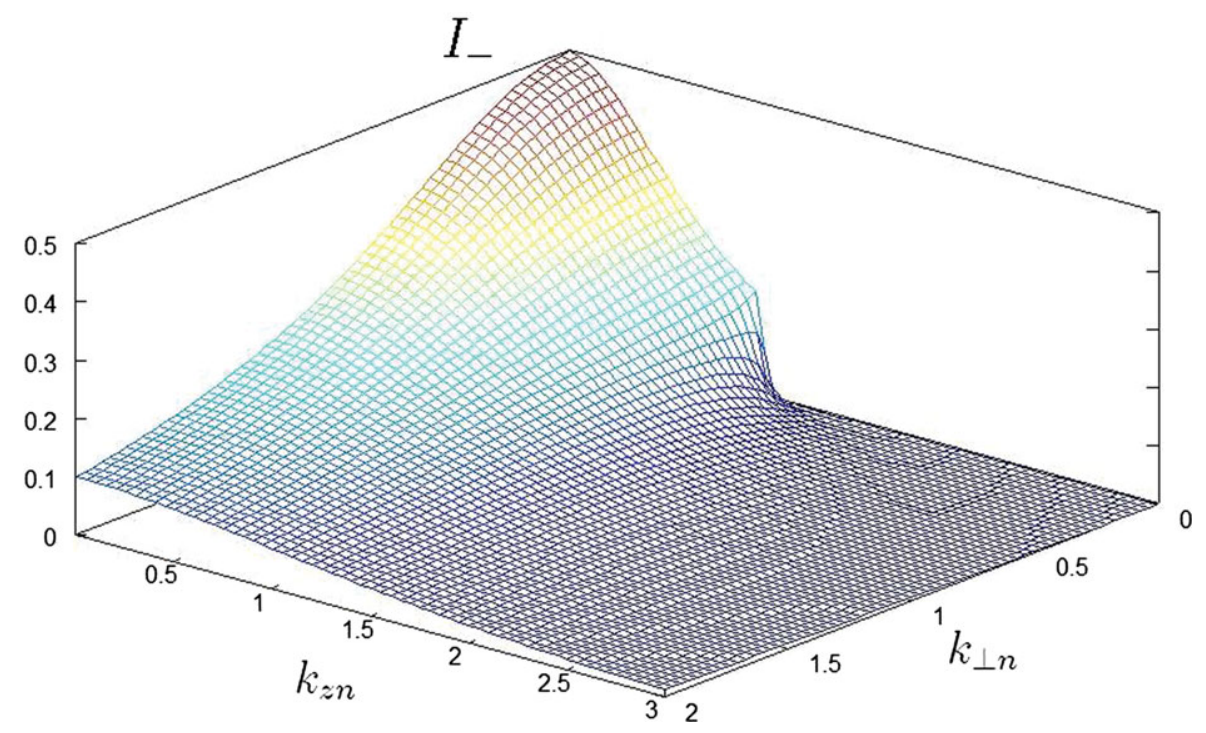

Figure 2. (Colour online) $I_{-}$plotted as a function of normalized wavenumbers $k_{z n}$ and $k_{\perp n}$.

that when $C_{\text {eff }}^{2}$ turns negative, short wavelength modes of the negative root will have the factor $\Omega_{i}^{2}+k^{2} C_{\mathrm{eff}}^{2}$ negative, in which case we always have an instability. The growth rate will then be of the order

$$
\gamma \sim k \frac{\left|u_{0}\right| \omega_{p i}}{c\left|2 \mathbf{k} \cdot \mathbf{k}_{0}-k^{2}\right|^{1 / 2}} .
$$

This expression for $\gamma$ can in principle be made very large since $2 \mathbf{k} \cdot \mathbf{k}_{0}-k^{2}$ can be adjusted to approach zero, but the validity of the expression breaks them down when $\left|2 \mathbf{k} \cdot \mathbf{k}_{0}-k^{2}\right|$ is of order $2 \gamma \omega_{0} / c^{2}$. The maximum growth rate $\gamma_{\max }$ is

$$
\gamma_{\max } \sim\left[k^{2} \frac{\left|u_{0}\right|^{2} \omega_{p i}^{2}}{\omega_{0}}\right]^{1 / 3} .
$$

However, we also require

$$
\gamma_{\max } \lesssim \frac{\left|u_{0}\right|^{2} \omega_{p i}^{2}}{C_{s}^{2} \omega_{0}}
$$

to keep $C_{\text {eff }}$ negative, which means that the maximum growth rate is limited by conditions (2.10) and (2.11). Since (2.10) can in principle be made arbitrarily large by increasing $k$, eventually the limit on the growth rate is guaranteed to be given by (2.11).

The transition from the parametric decay instability with a growth rate given by (2.5) to the large amplitude regime depends on the dimensionless amplitude $u_{0 n}=$ $\left|u_{0}\right| \omega_{p i} / C_{s}\left(\omega_{0} \Omega_{i}\right)^{1 / 2}$. In the regime $u_{0 n} \ll 1$, the growth rate scales as $\gamma / \Omega_{i} \sim u_{0 n}$, whereas for $u_{0 n}>1$, the scaling changes to $\gamma / \Omega_{i} \sim u_{0 n}^{2}$. The transition to a higher pump amplitude is accompanied by a shift in the wavelength of the low-frequency mode, i.e. from long to short wavelengths as compared to $C_{s} / \Omega_{i}$. For the large amplitude results to be valid, the amplitude must still be small enough for (1.1) to apply, which implies $\left|u_{0}\right|<\omega_{0} / k_{0}$. If this condition is violated the spectrum of the decay products will be more complicated than what we have considered here. In conclusion, there is thus a range of pump velocity amplitudes $C_{s}\left(\omega_{0} \Omega_{i}\right)^{1 / 2} / \omega_{p i}<\left|u_{0}\right|<\omega_{0} / k_{0}$ in which case the large amplitude scaling $\gamma / \Omega_{i} \sim u_{0 n}^{2}$ holds.

In order to compare with previous work, we finally consider the one-dimensional limit and then also include the contributions from both sidebands. The expression (2.7) accordingly turns out to be

$$
C_{\mathrm{eff}}^{2}=C_{s}^{2}-\frac{2\left|u_{0}^{2}\right| \omega_{p i}^{2}}{\left(k^{2}-4 k_{0}^{2}\right) c^{2}}
$$

which is in agreement with the previous result (Stenflo 1978).

About 40 years ago, most space physicists could not imagine stimulated Brillouin scattering in the ionosphere. However, although very weak, this effect finally turned out to exist (see the review paper by Stenflo 2004). Recently, it was suggested (Sharma et al. 2011) that stimulated Brillouin scattering also may exist in biological tissues. According to the mobile telephone industry, this effect this time also is expected to be negligible. However, due to unexpected resonance phenomena we cannot be sure. More studies are thus necessary.

\section{References}

Amin, M. R. 2010 J. Appl. Phys. 107, 023307.

Drake, J. F. et al. 1974 Phys. Fluids 17, 778.

Gurevich, A. V. 2007 Phys. Uspekhi 50, 1091.

Kruer, W. 1973 The Physics of Laser Plasma Interactions. Redwood City, CA: Addison-Wesley.

Mendonca, J. T. 2012 Plasma Phys. Contr. Fusion 54, 124031.

Panwar, A. and Sharma, A. K. 2009 J. Appl. Phys. 106, 063301.

Rahman, H. U., Rizvi, S. A. H., Yu, M. Y. and Shukla, P. K. 1981 Phys. Lett. A 84, 481.

Samimi, A. et al. 2013 J. Geophys. Res. 118, 502. 
Sharma, R. P., Batra, K. and Excell, P. S. 2011 J. Plasma Phys. 77, 117.

Shukla, P. K. and Tagare, S. G. 1979 J. Geophys. Res. 84, 1317. Shukla, P. K. and Stenflo, L. 2010 J. Plasma Phys. 76, 853.

Shukla, P. K., Yu, M. Y. and El-Nadi, A. 1984 Phys. Lett. A 106, 169.

Simon, A. 1995 Phys. Plasmas 2, 3832.

Stenflo, L. 1978 Phys. Scripta 17, 9.
Stenflo, L. 1981 Phys. Rev. A 23, 2730.

Stenflo, L. 2004 Phys. Scripta T 107, 262.

Tsytovich, V. N. et al. 1973 Phys. Scripta 7, 241.

Weiland, J. and Wilhelmsson, H. 1977 Coherent Nonlinear Interaction of Waves in Plasmas. New York, NY: Pergamon Press.

Yadav, S., Kaur, S. and Tripathi, V. K. 2008 Phys. Scripta 78, 065501 . 